\title{
Caracterización química y toxicológica del garbancillo (Astragalus garbancillo Cav.)
}

Chemical and toxicological characterization of Garbancillo (Astragalus garbancillo Cav.)

Juan Roque González ${ }^{1}$, Edson Yupanqui Torres ${ }^{2}$, Edell Aliaga Zegarra ${ }^{2}$, Jenny Álunarez Bautista ${ }^{1}$ y Olivio Castro Mandujano ${ }^{3}$

\section{RESUMEN}

El objetivo de la investigación es conocer los componentes químicos y tóxicos del garbancillo (Astrágalus garbancillo Cav.), para determinar los elementos activos tóxicos y adictivos de esta planta que provoca la muerte de los animales que lo consumen en el periodo de estiaje, ocasionando pérdidas económicas en la ganadería andina. Se ubicaron los lugares de toma de muestra considerando su abundancia: Huacacorral, distrito de Chiquián, provincia de Bolognesi; Kallan Punta, distrito de Independencia, provincia de Huaraz; Conococha, distrito de Pampas Chico, provincia de Recuay; distrito de San Marcos, provincia de Huari; todos ubicados en el departamento de Ancash. Los análisis a los que se sometieron las muestras son: análisis fitoquímico, determinación de ácidos grasos y esteroles (Cromatografía de gases), determinación de metales (método de ICP), determinación de selenio (absorción atómica con generación de hidruros), y la toxicidad. Entre los componentes de mayor presencia encontramos, ácidos grasos: palmítico (13,2\%) y esteárico (10,6\%). Esteroles: brassicasterol (44,03\%), $\beta$-sitosterol (22,3\%); estigmasterol (13,1\%). Metales mayoritarios: K (15 g/Kg), Ca (9,65 g/Kg), $\mathrm{Mg}(2,07 \mathrm{~g} / \mathrm{Kg})$, Se $(2,2 \mathrm{mg} / \mathrm{Kg})$. Fitoquímico: alcaloides, taninos, fenoles, esteroides y triterpenos $(+++)$ y la toxicidad como $\mathrm{DL}_{50}$ es mayor a $5,0 \mathrm{~g}$ de producto $/ \mathrm{Kg}$ de $\mathrm{pc}$, cantidad equivalente a $0,011 \mathrm{mg} \mathrm{Se} / \mathrm{Kg}$ de pc. La adicción del ganado a esta planta se debe a los alcaloides y la morbi-mortalidad al selenio, el garbancillo es un acumulador de selenio y su ingesta en cantidades abundantes lo hace tóxico.

Palabras clave: Astragalus garbancillo Cav; toxicidad; análisis químico.

\footnotetext{
1 Universidad Nacional «Santiago Antúnez de Mayolo». Huaraz, Perú.

2 Universidad Privada «Arzobispo Loayza». Lima, Perú.

3 Universidad Privada «Pontificia Universidad Católica del Perú». Lima, Perú.
} 


\section{ABSTRACT}

The aim of the research is to understand the chemical and toxic components garbancillo (Astragalus garbancillo Cav.), to learn about its addictive and toxic active components of this plant that causes the death of the animals that consume it during the dry season, causing economic losses in the Andean farming. To achieve the objective were places of specimen considering their wealth, these being the following: Huacacorral, Chiquián, Bolognesi province, Kallan district, district of Independencia, Huaraz province Conococha, district of Pampas Chico, province of Recuay, district of San Marcos, province of Huari, all located in the Department of Ancash. Analysis samples were subjected to are: analysis phytochemical (Dr. Olga Lock method), determination of fatty acids and sterols (gas chromatography), determination of metals (ICP method), determination of selenium (atomic absorption with hydride generation), and as $\mathrm{DL}_{50}$ toxicity (OECD Guideline 401 for testing of chemicals.) Acute oral toxicity). Greater presence components include, fatty acids: Palmitic (13, 2\%) and stearic (10, $6 \%$ ). Sterols: brassicasterol (44, 03\%), $\beta$-sitosterol (22, 3\%); stigmasterol (13, 1\%). Major metals: K (15 g/Kg), Ca (9, 65 g/Kg), Mg (2, 07 g/Kg), Se (2, 2 mg/Kg). Phytochemical: alkaloids, tannins, phenols, steroids and triterpenes $(+++)$ and toxicity as ${ }_{50}$ $\mathrm{DL}$ is greater than 5,0 g of product $/ \mathrm{Kg}$ pc, amount equivalent $0,011 \mathrm{mg} \mathrm{Se} / \mathrm{Kg}$ pc. Addiction of livestock this plant is due to the alkaloids and the morbidity and mortality to the Se, the garbancillo is an accumulator of is and their intake in abundant quantities makes it toxic.

Keywords: Astragalus garbancillo Cav:; toxicity; chemical analisis.

\section{ICHIKLLACHAW}

Kay musyapakuyqa kimikunapa, garwansillupa miyunta riqinaparaykurqmi rurakashqa, Kay qurapa miyunkuna riqinapaqraykurmi rurakashqa, kayqa ashmakunapa wanuynintam ashin usya patsachaw mikuyanqanchaw, kayqa manam allitsu antichaw uushakuna waataqkunapaq, kayta musyaytaqa rurakashqa Wankakanchachaw, Chikyan ichik suyuchaw; Kallan punta Waraspa ichik suyuchaw; Quñuqucha Riqway ichik suyuchaw; San Markus Wari ichik suyuchaw; kay llapan ichik llaqtakuna Anqash suyuchawmi kayan. Kaykuna analisakashqa: fitukimiku analisis, puchquq wirakunawan istirulis nishqanwan, chuqikuna kaynin, siliniyu kanqan, hinaman miyu $\mathrm{DL}_{50}$. Atska kaq kaqninkuna tarintsik, puchquq wirakuna: palmitiku $(13,2 \%)$ niykur istiyariku $(10,6 \%)$. Istirulis: brassikastirul (44,03\%), ß-situstirul (22,3\%); istigmastirul (13,1\%). Atska kaq chuqikuna: K (15 g/Kg), Ca (9,65 g/Kg), Mg (2,07 g/Kg), Se (2,2 mg/Kg). Fitukimiku: alkaluydis, taninus, finulis, istiruydis, tritirpinus $\left(+++\right.$ ) niykur miyukuna $\mathrm{DL}_{50}$ atskayan 5,0 $\mathrm{g}$ wachaynin/Kg pcpa, kayqa tinkun $0,011 \mathrm{mg} \mathrm{Se} / \mathrm{Kg}$ pckama. Kay plantapa uushankunapa yapaynin alkaluydikunawan murbi-wanuyninta, garbasilluqa silinuyu aylluyninmi, kaypa atska mikuyninqa miyuyaatsinmi.

Pushaq shimikuna: Astragalus garbancillu Cav, toxisidad, kimiku alli qawapashqa. 


\section{INTRODUCCIÓN}

La presente investigación contribuye al mejor conocimiento químico y toxicológico de esta planta, para adoptar medidas preventivas la intoxicación del ganado evitan así las pérdidas económicas que afectan a los ganaderos por la morbi-mortalidad de sus animales.

El Astragalus garbancillo Cav., o simplemente «garbancillo» es una leguminosa silvestre que crece en las praderas nativas de la zona altoandina entre los 2700 a 4500 msnm. Cuando la pradera está sobrepastoreada y en estiaje se comporta como una planta invasora y el ganado la consume; la ingesta con el tiempo genera un proceso de intoxicación que termina con una afección clara al sistema nervioso en las primeras etapas y la muerte en las fases avanzadas, lo cual hace inviable el consumo de la carne del animal muerto.

Los equinos, ovinos y vacunos son las especies más susceptibles. Los animales que las consumen se vuelven adictos y cuando lo hacen por mucho tiempo mueren. Los animales intoxicados presentan pelo anormalmente largo, depresión, los nervios ópticos son afectados, pérdida del control muscular, temblores en los miembros anteriores; conforme la intoxicación avanza el animal enflaquece y deja de comer, sobreviene una parálisis total y muere. Este proceso dura aproximadamente dos meses. En los primeros cinco días de consumo, el animal se puede recuperar con un tratamiento adecuado o con el retiro total del garbancillo de su dieta.

El presente estudio ha permitido conocer la composición química y toxicológica de esta planta, según lo cual los metabolitos que se encuentran en mayor cantidad $(+++)$ son los taninos, fenoles, alcaloides, esteroides y triterpenos; en cantidad media $(++)$ los flavonoides y en menor cuantía (+) las quinonas y catequinas. Los alcaloides son las sustancias responsables de generar adicción en los animales. Entre los metales presentes en el garbancillo sobresale el alto contenido de selenio $(2,2 \mathrm{mg} / \mathrm{kg})$, siendo este valor muy alto con respecto a otras especies forrajeras $(<0,1 \mathrm{mg} / \mathrm{kg})$. Según normas internacionales el contenido máximo permisible de selenio en una planta forrajera es de 0,2 mg/kg. Según NRC (2001), el consumo máximo recomendable es de $6 \mathrm{mg} /$ vaca/día. Entonces, una vaca de $350 \mathrm{~kg}$ de peso vivo, consumiendo garbancillo en el equivalente al $50 \%$ de su requerimiento de forraje verde, ingeriría aproximadamente $38 \mathrm{mg}$ de Se/día. Se acepta que, el selenio es el responsable de la intoxicación en el ganado. La prueba de toxicidad en ratas, no produjo mortalidad en las cuatro dosis del ensayo. Por lo tanto, la $\mathrm{DL}_{50}$ es mayor de 5,0 g de producto $/ \mathrm{Kg}$ pc de rata.

\section{MATERIALES Y MÉTODOS}

La presente investigación es básica y descriptiva. El diseño de investigación seleccionado es no experimental cuantitativo.

La parte aérea del garbancillo (hojas y ramas delgadas recolectadas) en los diferentes puntos de muestreo se sometió a un lavado, secado bajo sombra, triturado, molido y enviada al laboratorio para los respectivos análisis. 
Análisis fitoquímico utilizando la marcha fitoquímica tradicional de Lock (1994).

Análisis de ácidos grasos y esteroles: se utilizó por cromatografía de gases, método de la AOAC. 199, utilizando el Cromatógrafo de gases, Hewlett Packard, 5890 serie II. Condiciones cromatográficas: Equipo autosystem PE, columna capilar DB-225(30m x $0,32 \mathrm{~mm} \times 0,25 \mu \mathrm{m})$, programación de temperatura de la columna $1^{\prime} / 70^{\circ} / 10^{\circ} /$ $\min / 180^{\circ} / 3^{\circ} / \mathrm{min} / 220^{\circ} / 5^{\prime}$, temperatura de inyección y detección $250^{\circ}$, detector FID, gas de arrastre helio, flujo $1 \mathrm{~mL} / \mathrm{min}$., volumen inyectado $1 \mu \mathrm{L}$, Split 1:100.

Análisis de metales por el método de espectroscopía de emisión por plasma inductivamente acoplado. Método EPA-200.7-rev. 4.4-1994, el equipo usado fue ICP/ PRE-130.

Análisis de selenio por el método de espectroscopía de absorción atómica por generación de hidruros. Método basado en SM (Standard Methods for Examination of Water\&Wastewater-2005) M 3114 B y C, el equipo utilizado fue un Perkin-Elmer 3110.

Determinación de toxicidad oral aguda, DL50 OECD Guideline 401 for testing of chemicals. Acute oral toxicity, recomendado por la Comunidad Andina; se utilizó ratas de laboratorio: 20 ratas albinas machos y 5 ratas control del mismo sexo de la cepa Holtzman.

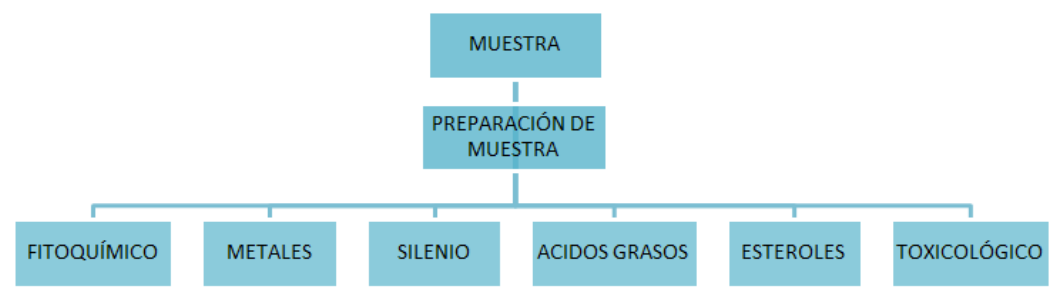

Figura 1. Flujo de evaluaciones del Astragalus garbancillo

\section{RESULTADOS}

En la tabla 1, se muestra los resultados del análisis fitoquímico realizado a plantas de garbancillo, notándose un alto contenido de taninos, fenoles, alcaloides, esteroides y triterpenos; en cantidad moderada flavonoides y en baja concentración quinonas y catequinas.

Tabla 1. Resultados del análisis fitoquímico

\begin{tabular}{llc}
\hline Reactivo & Compuesto encontrado & Cantidad \\
\hline $\mathrm{FeCl}_{3}$ & Taninos y fenoles & +++ \\
Borntrager & Quinonas & + \\
Shinoda & Flavonoides & ++ \\
Rosenheim & Catequinas & + \\
Mayer & & \\
Dragendorff & Alcaloides & +++ \\
Wagner & & \\
Lieberman & Esteroides & +++ \\
Bouchard & Triterpenos & +++ \\
\hline
\end{tabular}


El análisis químico para determinar la cantidad de metales presentes en plantas de garbancillo se reporta en la tabla 2. Se observa un alto contenido de potasio $(15 \mathrm{~g} / \mathrm{kg})$, calcio $(9,6 \mathrm{~g} / \mathrm{kg})$, magnesio $(2 \mathrm{~g} / \mathrm{kg})$, hierro $(0,4 \mathrm{~g} / \mathrm{kg})$ y aluminio $(0,25 \mathrm{~g} / \mathrm{kg})$. Cabe resaltar el alto contenido de selenio $(2,2 \mathrm{mg} / \mathrm{kg})$.

Tabla 2. Contenido de metales

\begin{tabular}{cccccc}
\hline Elemento & $\begin{array}{c}\text { Cantidad } \\
(\mathrm{mg} / \mathrm{Kg})\end{array}$ & Elemento & $\begin{array}{c}\text { Cantidad } \\
(\mathrm{mg} / \mathrm{Kg})\end{array}$ & Elemento & $\begin{array}{c}\text { Cantidad } \\
(\mathrm{mg} / \mathrm{Kg})\end{array}$ \\
\hline $\mathrm{K}$ & 14982,0 & $\mathrm{Zn}$ & 22,6 & $\mathrm{Sn}$ & 0,6 \\
$\mathrm{Ca}$ & 9654,0 & $\mathrm{Cu}$ & 6,3 & $\mathrm{Ag}$ & 0,5 \\
$\mathrm{Mg}$ & 2070,0 & $\mathrm{Ti}$ & 3,9 & $\mathrm{Tl}$ & 0,5 \\
$\mathrm{Fe}$ & 371,1 & $\mathrm{Ba}$ & 3,6 & $\mathrm{Co}$ & 0,3 \\
$\mathrm{Al}$ & 249,0 & $\mathrm{Se}$ & 2,2 & $\mathrm{Mo}$ & 0,3 \\
$\mathrm{Mn}$ & 68,7 & $\mathrm{Cr}$ & 2,2 & $\mathrm{Cd}$ & 0,1 \\
$\mathrm{Sr}$ & 48,1 & $\mathrm{Ni}$ & 0,7 & $\mathrm{Be}$ & 0,1 \\
$\mathrm{Na}$ & 47,4 & $\mathrm{~Pb}$ & 0,6 & $\mathrm{~V}$ & 0,6 \\
$\mathrm{~B}$ & 23,0 & & & & \\
\hline
\end{tabular}

Los ácidos grasos encontrados en la planta son: Ácido esteárico (10,6\%), Ácido cis-oleico (6,7\%), Ácido linoleico (5,8\%) y Ácido palmítico (3,2\%). Ver tabla 3.

Tabla 3. Contenido de ácidos grasos

\begin{tabular}{lc}
\hline Ácidos grasos & \% relativo \\
\hline Ácido esteárico (18:0) & 10,6 \\
Ácido cis-oleico (18:1) & 6,7 \\
Ácido linoleico (18:2) & 5,8 \\
Ácido palmítico (16:0) & 3,2 \\
\hline
\end{tabular}

En la tabla 4, se reporta la cantidad de esteroles hallados en muestras de garbancillo; así tenemos: Brassicasterol (44,03\%), $\beta$-sitosterol (22,3\%) y estigmasterol $(13,1 \%)$.

Tabla 4. Resultado del análisis de esteroles

\begin{tabular}{lc}
\hline Esteroles & $\%$ relativo \\
\hline Brassicasterol & 44,03 \\
$\beta$-sitosterol & 22,30 \\
Stigmasterol & 13,10 \\
\hline
\end{tabular}

La prueba de toxicidad realizada en ratas, no produjo mortalidad en las cuatro dosis del ensayo, tal como se puede observar en la tabla 5. 
Tabla 5. Toxicidad aguda del garbancillo

\begin{tabular}{cc}
\hline $\begin{array}{c}\text { Dosis } \\
\text { (g/kg rata) }\end{array}$ & $\begin{array}{c}\text { Ratas muertas } \\
\text { Total }\end{array}$ \\
\hline 5,0 & $0 / 5$ \\
2,5 & $0 / 5$ \\
1,25 & $0 / 5$ \\
0,625 & $0 / 5$ \\
Control & $0 / 5$ \\
\hline
\end{tabular}

\section{DISCUSIÓN}

Según el análisis fitoquímico los metabolitos mayoritarios (+++) en el Astragalus garbancillo son: los taninos y fenoles, alcaloides, esteroides y triterpenos; en cantidad media $(++)$ los flavonoides. De estos metabolitos nos interesan los alcaloides porque son los responsables de generar adicción al garbancillo en los animales. Según Aguadé y Chávez (2014) la locaína es el componente tóxico en el garbancillo.

El garbancillo tiene altos contenidos de los siguientes metales: potasio $(15 \mathrm{~g} / \mathrm{kg})$, seguido del calcio $(9,6 \mathrm{~g} / \mathrm{kg})$, magnesio $(2 \mathrm{~g} / \mathrm{kg})$, hierro $(0,4 \mathrm{~g} / \mathrm{kg})$ y aluminio $(0,25 \mathrm{~g} / \mathrm{kg})$. Por tanto, esta planta es una buena fuente de potasio, calcio y magnesio, pero el hecho de que tiene otros elementos tóxicos, hace peligroso su consumo por los animales.

Según Brack (1999), el garbancillo es una planta acumuladora de selenio, el contenido de selenio determinado en el garbancillo es de $2,2 \mathrm{mg} / \mathrm{kg}$, siendo este valor muy alto comparado con el de otras especies forrajeras $(<0,1 \mathrm{mg} / \mathrm{kg})$. Según normas internacionales el contenido máximo permisible de selenio en una planta forrajera es de 0,2 mg/kg. Según National Research Council (2001), el consumo máximo recomendable es de $6 \mathrm{mg} / \mathrm{vaca} /$ día. Una vaca de $350 \mathrm{~kg}$ de peso vivo, consumiendo garbancillo en el equivalente al 50\% de su requerimiento de forraje verde, ingeriría aproximadamente $38 \mathrm{mg}$ de Se/día. Si bien, es cierto que el selenio es necesario en cantidades pequeñas en la alimentación de los animales, porque es antioxidante, en cantidades altas es tóxico, su límite máximo tolerable en la alimentación es de $2 \mathrm{mg} \mathrm{Se} / \mathrm{kg}$ de alimento y el nivel tóxico es de $8 \mathrm{mg} / \mathrm{Kg}$ (National Research Council, 2001) y tener en cuenta que este elemento es acumulable. Entonces, el selenio es el que genera las lesiones histopatológicas, con sintomatología típica en el ganado que lo consume, llevándolo hasta la muerte. Según Aguadé y Chávez (2014) las plantas que tienen niveles tóxicos de selenio, producen envenenamientos agudos y crónicos en el ganado que las consumen. Los primeros son poco frecuentes, mientras que la intoxicación crónica produce ceguera, incoordinación y la llamada «enfermedad alcalina».

Los ácidos grasos encontrados en la planta son: ácido esteárico (10,6\%), ácido cis-oleico $(6,7 \%)$, ácido linoleico $(5,8 \%)$ y ácido palmítico (3,2\%). No se ha encontrado ningún ácido graso del tipo omega 3 u omega 6. Los ácidos esteárico y palmítico son saturados, mientras que el cis-oleico y el linoleico son insaturados.Los ácidos grasos insaturados son beneficiosos en la salud del ser humano, porque disminuyen el riesgo de enfermedades cardiacas coronarias. 
Los esteroles o fitoesteroles hallados en el garbancillo son: Brassicasterol (44,03\%), $\beta$-sitosterol (22,3\%) y estigmasterol $(13,1 \%)$, cuyos valores aparentemente no generan efecto nocivo en los animales; por el contrario, ayudan a mejorar la eficiencia reproductiva de los animales y tienen propiedades antioxidantes, su consumo es beneficioso en la salud humana y fortalecen el sistema inmune.

La prueba de toxicidad en ratas no produjo mortalidad en las cuatro dosis del ensayo. Por lo tanto, la $\mathrm{DL}_{50}$ por vía oral de la muestra diluida en agua destilada es mayor a 5,0 $\mathrm{g}$ de producto $/ \mathrm{Kg}$ peso corporal de rata; esta cantidad equivale a 0,011 $\mathrm{mg} \mathrm{Se} / \mathrm{Kg}$ de pc, y el nivel tóxico es de $8 \mathrm{mg} \mathrm{Se} / \mathrm{Kg}$ de producto. Siendo el selenio acumulativo en los seres vivos, la ingesta de garbancillo en cantidades abundantes lo hace tóxico.

\section{CONCLUSIONES}

Según el análisis fitoquímico, los metabolitos que se encuentran en mayor cantidad $(+++)$ en el Astragalus garbancillo son: los taninos y fenoles, alcaloides, esteroides y triterpenos; en cantidad media $(++)$ los flavonoides y en menor cuantía $(+)$ las quinonas y catequinas. Los alcaloides son las sustancias responsables de generar adicción en los animales (locaína).

El contenido de metales en el garbancillo es como sigue: potasio $(15 \mathrm{~g} / \mathrm{kg})$, seguido del calcio $(9,6 \mathrm{~g} / \mathrm{kg})$, magnesio $(2 \mathrm{~g} / \mathrm{kg})$, hierro $(0,4 \mathrm{~g} / \mathrm{kg})$ y aluminio $(0,25 \mathrm{~g} / \mathrm{kg})$, y los demás metales tienen valores entre 0,1 a $69 \mathrm{mg} / \mathrm{kg}$. Esta planta es una buena fuente de potasio, calcio y magnesio.

El contenido de selenio en el garbancillo es de $2,2 \mathrm{mg} / \mathrm{kg}$, siendo este valor muy alto con respecto a otras especies forrajeras $(<0,1 \mathrm{mg} / \mathrm{kg})$. Según normas internacionales el contenido máximo permisible de selenio en una planta forrajera es de $0,2 \mathrm{mg} / \mathrm{kg}$. Según NRC (2001), el consumo máximo recomendable es de $6 \mathrm{mg} / \mathrm{vaca} /$ día. Una vaca de $350 \mathrm{~kg}$ de peso vivo, consumiendo garbancillo en el equivalente al 50\% de su requerimiento de forraje verde, ingeriría aproximadamente $38 \mathrm{mg}$ de Se/día. Entonces, el selenio es el responsable de la intoxicación en el ganado.

Los ácidos grasos encontrados en la planta son: Acido esteárico (10,6\%), Acido cis-oleico (6,7\%), Acido linoleico (5,8\%) y Acido palmítico (3,2\%). Los ácidos esteárico y palmítico son saturados, mientras que el cis-oleico y el linoleico son insaturados.

Los esteroles hallados en el garbancillo son: Brassicasterol (44,03\%), $\beta$-sitosterol $(22,3 \%)$ y estigmasterol $(13,1 \%)$; estos no son nocivos para los animales; por el contrario son beneficiosos.

La prueba de toxicidad en ratas no produjo mortalidad en las cuatro dosis del ensayo. Por lo tanto, la $\mathrm{DL}_{50}$ es mayor de $5,0 \mathrm{~g}$ de producto $/ \mathrm{Kg}$ pc de rata. 
| Juan Roque, Edson Yupanqui, Edell Aliaga, Jenny Álvarez y Olivio Castro

\section{REFERENCIAS BIBLIOGRÁFICAS}

Aguadé, Paul y Chávez, Juan. 2014. Plantas tóxicas para el ganado: su identificación, manejo y control. Xochimilco: Universidad Autónoma Metropolitana.

Brack, Antonio. 1999. Diccionario enciclopédico de plantas útiles del Perú. Cusco: Ed. CBC.

Lock de Ugaz, Olga. 1994. Investigación fitoquímica. Métodos en el estudio de productos naturales. Lima: Ed. Fondo Editorial-PUCP. 2da. Ed.

NATIONAL RESEARCH COUNCIL (NRC). 2001. Nutrient Requirements of Dairy Cattle 7 ma. Washington: Ed. National Academy Press.

Fecha de recepción: 29 de enero 2016

Fecha de aceptación: 30 de mayo 2016

\section{Correspondencia}

Juan Moisés Roque González

jmrg5556@cip.org.pe

\section{ANEXOS: FOTOGRAFÍAS}

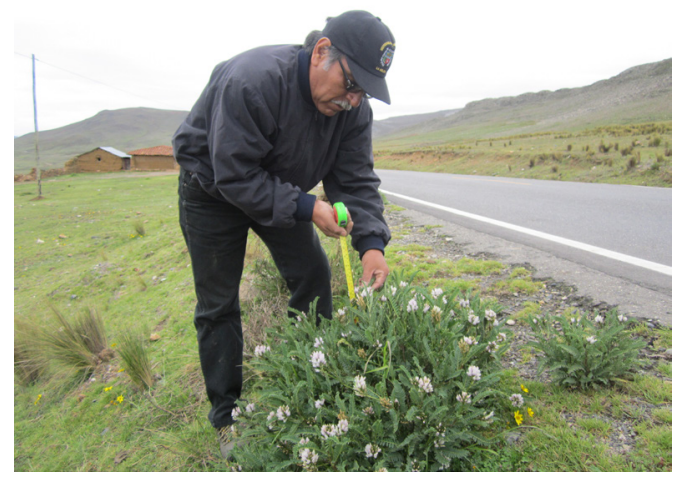

Figura 1. Evaluación biométrica de la planta

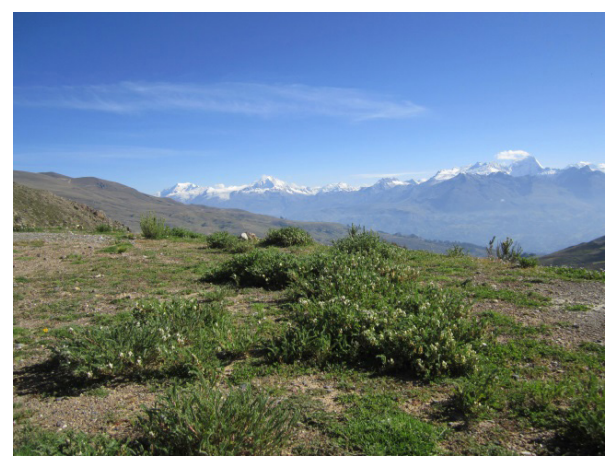

Figura 3. Comunidad de garbancillo en Ayash, San marcos-Huari

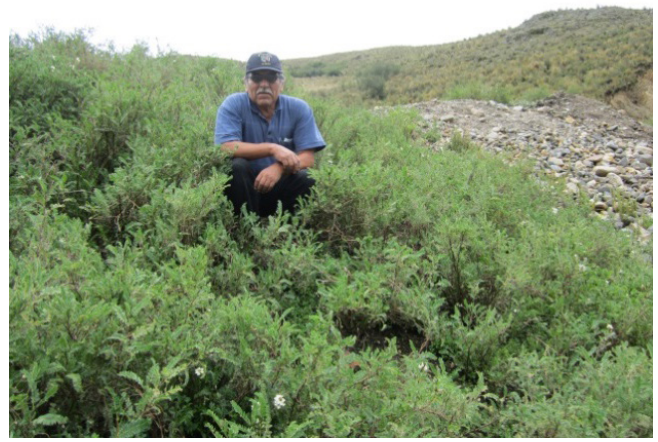

Figura 2. Garbancillo en Kallan punta $-\mathrm{Hz}$

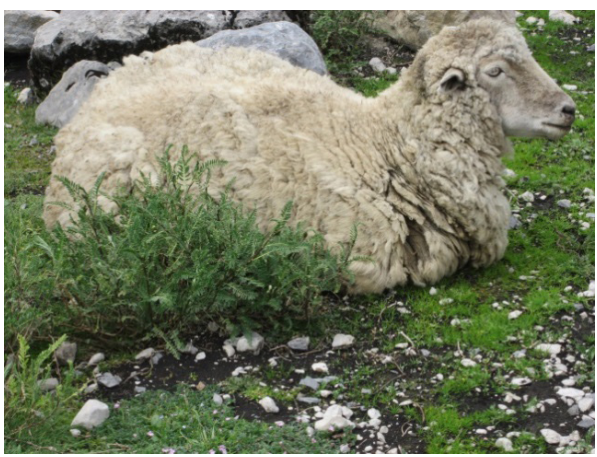

Figura 4. Ovino, luego de haber consumido garbancillo 